\title{
Community treatment orders: current evidence and the implications
}

\author{
Jorun Rugkåsa and John Dawson
}

\begin{abstract}
Summary
Community treatment orders (CTOS) have been widely introduced to address the problems faced by 'revolving door' patients. A number of case-control studies have been conducted but show conflicting results concerning the effectiveness of CTOs. The Oxford Community Treatment Order Evaluation Trial (OCTET) is the third randomised controlled trial (RCT) to show that CTOs do not reduce rates of readmission over 12 months,
\end{abstract}

despite restricting patients' autonomy. This evidence gives pause for thought about current CTO practice. Further high-quality RCTs may settle the contentious debate about effectiveness.

\section{Declaration of interest}

J.R. and J.D. were the director and an international advisor respectively of the OCTET study.
Following widespread deinstitutionalisation of psychiatric services, community treatment orders (CTOs, also known as mandated out-patient treatment, out-patient commitment, assisted out-patient treatment and supervised community treatment) have been introduced in many Western jurisdictions to authorise extended periods of compulsory community care. This has shifted compulsion in hospital away from the centre of mental health law. A CTO requires the patient to adhere to out-patient treatment. The aim is to help patients achieve stability, to control risk and to provide less restrictive alternatives to (repeated) involuntary admission to hospital. ${ }^{1}$ The main mechanism of enforcement is swift recall to hospital for treatment when required. We reflect on current evidence for CTO effectiveness and implications for practice.

\section{The spread and use of CTOs}

Community treatment orders are established in over 70 jurisdictions. They have existed since the 1980s or early 1990s in most parts of North America and Australasia., ${ }^{1,2}$ More recently they have been introduced in England and Wales, Norway, Israel, Scotland, Sweden and Switzerland. The legal regimes vary significantly in detail, including whether patients with capacity who object can go on a CTO, the need for prior hospital admissions and the scope of powers conferred over out-patients.

The rate of use of CTOs varies widely between and within jurisdictions. They tend to be used more frequently in Australasia than in North America, with European jurisdictions falling in-between. Within a single jurisdiction, use often varies considerably between regions and individual psychiatrists. It has been suggested that when CTOs are introduced they increase the overall level of coercion in psychiatry. ${ }^{2}$

Community treatment orders are often contentious. They generally garner support from families of those with a serious mental illness but meet strong opposition from patient advocates. The views among clinicians are often divided. ${ }^{3}$ Opposition has commonly focused on the patient's right not to have their autonomy restricted rather than on their right to receive adequate care. Recently, the focus of debate has shifted to evidence of CTO effectiveness. ${ }^{3,4}$

\section{What does the current evidence say?}

Community treatment order laws do not explicitly state the standards to be used to measure their effectiveness. Various measures of hospital use are the most common outcomes applied in published studies. Frequency of contact with out-patient services, victimisation, arrest, mortality and quality of life measures have been investigated in some studies. Literature reviews have not established that CTOs confer advantages for patients. ${ }^{1,2,5}$ The research provides three broad levels of evidence: descriptive studies; case-control studies, involving matched pairs or 'before and after' designs; and randomised controlled trials (RCTs).

\section{Descriptive studies}

Descriptive studies consist largely of local investigations of CTO patient populations or stakeholder views and include qualitative studies of personal experiences. Many report reductions in readmission rates and improved stability after a number of months. However, the study designs used mean the changes observed cannot confidently be attributed to CTOs. The studies do indicate that the groups of patients on CTOs are remarkably similar across jurisdictions. Patients on CTOs tend to be male, in middle age, diagnosed with schizophrenia, with several prior hospital admissions and a history of non-adherence. Many misuse substances, are single, self-neglecting and relatively isolated. ${ }^{1}$ This shows that clinicians who use CTOs have similar ideas about whom they think will benefit.

\section{Case-control studies}

A number of case-control studies have been conducted, using either matched controls (in controlled before and after (CBA) studies) or patients serving as their own controls (uncontrolled before and after (UBA) studies). These studies report discrepant results regarding outcomes relating to admissions to hospital and community tenure. Most report increases in follow-up with out-patient services and shorter readmissions for patients on CTOs.

Studies of this kind are vulnerable to confounding influences, such as changes that may occur over time in the availability of beds, specialist teams or case management. ${ }^{4}$ Moreover, CBA studies may be confounded by difficulties in matching key patient characteristics such as lack of insight or adherence. The benefits reported by UBA studies may be affected by regression to the mean as the patient may recover regardless of being on a CTO because of the naturally fluctuating course of their condition. ${ }^{4}$ Studies using large available data-sets commonly test numerous hypothesised associations, which increases the likelihood of false 
positives. ${ }^{6}$ It is often unclear whether any measures (for example Bonferroni corrections or data splitting ${ }^{7}$ ) have been applied to avoid such problems.

Two large data-sets, one in Victoria, Australia, and another in New York, USA, have been used in a number of studies. The Victoria studies largely report increases in readmission rates, ${ }^{8,9}$ whereas the New York studies generally find reductions. ${ }^{10} \mathrm{~A}$ possible explanation for reduction in readmissions, where observed, is deliberate focusing of extra mental health services on patients on CTOs. If more intensive treatment is offered to patients on CTOs, these studies cannot distinguish with confidence whether the element of compulsion has an independent effect. In the service system recently studied in New York, for instance, assertive outreach, including case management (known to increase patient engagement) ${ }^{11}$ was prioritised to those on CTOs. ${ }^{10}$ In one Toronto-based study, however, where equal access was provided to case management, better outcomes were found for patients on CTOs. ${ }^{12}$

A number of studies report improved outcomes when CTOs are sustained for more than 6 months. ${ }^{10,12-14}$ However, selection bias may affect the outcome of these studies if only patients for whom the CTO seems to be working are kept on the order long term. ${ }^{15}$

In brief, the conflicting results and methodological limitations of the case-control studies make it difficult to draw conclusions about CTO effectiveness from this body of evidence as a whole.

\section{RCTS}

Randomised controlled trials provide the most rigorous test of treatment effect. They reduce the possible effects of sample bias and regression to the mean and they identify in advance both the hypothesis and the outcome measures to be used. Conducting RCTs of CTOs is challenging for ethical and legal reasons. ${ }^{16}$ Only three have been conducted, two in the USA 15 years ago ${ }^{17,18}$ and one recently in England (the Oxford Community Treatment Order Evaluation Trial, OCTET) ${ }^{19}$ Treatment under the CTO was compared with voluntary status (in the trials in the USA) and with discharge via a brief period of hospital leave in preparation for voluntary status (in OCTET) in England. All three trials report that CTOs did not reduce readmission rates over 11-12 months compared with the control condition. Moreover, on time to readmission, total days in hospital, psychiatric symptoms or global functioning, no difference was found in these trials. Patients received case management and close clinical monitoring in both arms of all three trials.

Randomised controlled trials have their own potential limitations, including refusal, attrition and protocol violations. The New York trial experienced considerable problems including a smaller than expected sample size $(n=142)$, high attrition and lack of adherence to the protocol, making it difficult to draw firm conclusions. The North Carolina trial $(n=264)$ had a refusal rate of $12 \%$ and an $18.2 \%$ attrition rate. The control group was 'immunised' from placement on a CTO during the follow-up year, and released if inadvertently placed on one. Patients with a history of violence were not randomised. In England, OCTET did not exclude participants on the basis of dangerousness. It had a refusal rate of $20 \%$. Only one of 336 patients withdrew and primary outcome data were obtained for all. It was impossible to control for protocol violations, ${ }^{16,19}$ and the treatment of around a quarter of the patients did not follow the randomised condition. A sensitivity analysis removing these patients did not alter the OCTET study findings. ${ }^{19}$

Aspects of each trial and differences between them may affect their generalisability. The US studies examined court-ordered
CTOs and randomised between CTO and voluntary status. OCTET studied clinician-initiated CTOs and randomised between discharge via CTO and via Section 17 leave under the Mental Health Act 1983 (the latter leading to significantly shorter periods under compulsion). The US studies actively excluded a proportion of patients, and OCTET and the New York trials experienced more protocol violations than expected. However, the clear differences in the length of coercion imposed on patients and the comparability of the treatment otherwise received in the two arms of these trials indicate that their common findings - no advantage to CTOs in readmission rates - are comparable and noteworthy. There remains a suggestion (echoing the findings in the CBA studies) that the duration of readmissions may be reduced by CTOs, although this seems variable and requires further testing.

\section{What are the implications?}

The current evidence from RCTs suggests that CTOs do not reduce readmission rates over 12 months. Seen in conjunction with the results of case-control studies, this may suggest it is intensive community services, not coercion, that positively affects hospital outcomes. When CTOs restrict patients' autonomy, however, and there is a duty to provide the least restrictive form of treatment, the results of the RCTs must give pause for thought. Settling uncertainties around CTOs will require further highquality RCTs. Optimum trials need mechanisms under which all eligible patients are randomised, a sufficient sample size, few protocol violations and a study period of sufficient duration to measure longer-term effects. It would be best to measure a wider range of outcomes, including the impact on the quality of life of some of our most vulnerable citizens.

Jorun Rugkåsa, PhD, Health Services Research Unit, Akershus University Hospital, Norway, and Department of Psychiatry, University of Oxford, UK; John Dawson, LLD, Faculty of Law, University of Otago, Dunedin, New Zealand

Correspondence: Jorun Rugkåsa, Health Services Research Unit, Akershus University Hospital, 1478 Lørenskog, Norway. Email: jorun.rugkasa@ahus.no First received 15 Jun 2013, accepted 5 Sep 2013

\section{References}

1 Churchill R, Owen G, Hotopf M, Singh S. International Experiences of Using Community Treatment Orders. Department of Health and Institute of Psychiatry, King's College London, 2007.

2 Dawson J. Community Treatment Orders: International Comparisons. University of Otago, 2005.

3 Burns T, Dawson J. Community treatment orders: how ethical without experimental evidence? Psychol Med 2009; 39: 1583-6.

4 O'Reilly R. Research on community treatment orders. In Applied Research and Evaluation in Community Mental Health Services (eds ER Vingilis, SA State): 68-79. McGill-Queen's University Press, 2009.

5 Kisely S, Campbell LA, Preston N. Compulsory community and involuntary outpatient treatment for patients with severe mental health disorders. Cochrane Database Syst Rev 2005; 3: CD004408.

6 Ioannis JP. Why most published research findings are false. PLOS Med 2005; 2: 696-701.

7 Dahl F, Grotle M, Benth JS, Natvig B. Data splitting as a countermeasure against hypothesis fishing: with a case study of predictors for low back pain. Eur J Epidemiol 2008; 23: 237-42.

8 Burgess P, Bindman J, Leese M, Henderson C, Szmukler G. Do community treatment orders for mental illness reduce readmission to hospital? An epidemiological study. Soc Psychiatry Psychiatr Epidemiol 2006; 41: 574-9.

9 Segal S, Burgess P. Conditional release, a less restrictive alternative to hospitalization? Psychiatr Serv 2006; 57: 1600-6.

10 Swartz M, Wilder C, Swanson J, Van Dorn R, Robbins $\mathrm{P}$, Steadman $\mathrm{H}$, et al Assessing outcomes for consumers in New York's assisted outpatient treatment program. Psychiatr Serv 2010; 61: 976-81. 
11 Killaspy $H$, Kingett $S$, Bebbington $P$, Blizard $R$, Johnson $S$, Nolan $F$, et al. Randomised evaluation of assertive community treatment: 3-year outcomes. Br J Psychiatry 2009; 195: 81-2.

12 Hunt $A$, da Silva A, Lurie S, Goldbloom D. Community treatment orders in Toronto: the emerging data. Can J Psychiatry 2007; 52: 647-55.

13 Segal S, Burgess P. The utility of extended outpatient civil commitment. Int J Law Psychiatry 2006; 29: 525-34.

14 Van Dorn R, Swanson J, Swartz M, Wilder C, Moser L, Gilbert A, et al Continuing medication and hospitalization outcomes after assisted outpatient treatment in New York. Psychiatr Serv 2010; 61: 982-7.

15 Szmukler G, Hotopf M. Effectiveness of involuntary outpatient commitment Am J Psychiatry 2001; 158: 653-4.
16 Dawson J, Burns T, Rugkåsa J. Lawfulness of a randomised trial of the new community treatment order regime for England and Wales. Med Law Rev 2011; 19: 1-26.

17 Swartz MS, Swanson JW, Wagner HR, Burns BJ, Hiday VA, Borum R. Can involuntary outpatient commitment reduce hospital recidivism? Findings from a randomized trial with severely mentally ill individuals. Am J Psychiatry 1999; 156: 1968-75.

18 Steadman H, Gounis K, Dennis D, Hopper K, Roche B, Swartz M, et al. Assessing the New York City involuntary outpatient commitment pilot programme. Psychiatr Serv 2001; 52: 330-6.

19 Burns T, Rugkåsa J, Molodynksi A, Dawson J, Yeeles K, Vazquez-Montes M, et al. Community treatment orders for patients with psychosis: a randomised controlled trial (OCTET). Lancet 2013; 381: 1627-33.

\section{psychiatry} in music

\section{Hip-hop and psychiatry: a fair rap?}

\section{Thomas James Reilly}

The link between rap and mental health is the promotion of harmful, self-destructive behaviour. The issue of drugs has been central to the gangster rap phenomenon. Alcohol abuse and casual sex are similarly ubiquitous in modern rap. Arguably, however, it is not much different from other musical styles such as rock or dance. The morals of hip-hop cannot be readily separated from the morals of the music industry.

Gangster rap's machismo is personified in Notorious B.I.G. who claimed to only know about 'money, hoes and clothes'. Behind the bravado though, he delivered insightful reflections on a great deal more. Biggie is a prime example of rap's ability to express mental vulnerability in an otherwise hyper-masculine culture. On the track Suicidal Thoughts he contemplates slitting his wrists and the real person behind the thug caricature is unveiled.

Hip-hop duo Gang Starr were among a group of artists keen to expose gangster rap clichés. Their 1998 hit Moment of Truth centres on reaching a nadir, deciding whether to live or die:

I'm ready to lose my mind but instead I use my mind/

I put down my knife and take the bullets out my nine.

These hip-hop luminaries are not ashamed to rap about suicide. Instead, they try to highlight that it takes strength to admit and overcome thoughts of self-harm.

British hip-hop has a similar image. Dizzee Rascal's debut Boy in Da Corner has all the usual hip-hop touchstones of sex, violence and drugs. The closing track Do it, though, is a personal admission of Dizzee's own depression:

Some days I wake up wishing I could sleep for good/

And if I had the guts to end it all believe I would

If you wanna get through it, stretch your mind to the limit/

You can do it.

Here, suicide is not a cowardly act but more akin to the Greco-Roman ideal of an honourable death, a death which takes courage. Nevertheless, he goes on to encourage listeners to find their own strength in overcoming mental difficulties. The message from hip-hop is that it is normal to feel depressed and it is not necessarily an admission of weakness. 